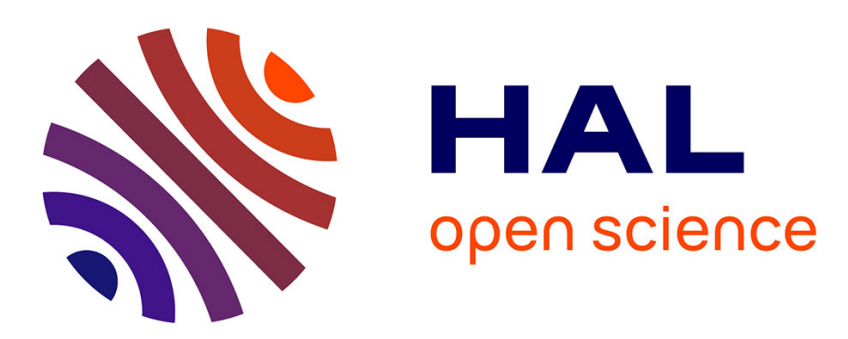

\title{
An error estimate for the approximation of linear parabolic equations by the Gradient Discretization Method
}

Jérôme Droniou, Robert Eymard, Thierry Gallouët, Cindy Guichard, Raphaele Herbin

\section{To cite this version:}

Jérôme Droniou, Robert Eymard, Thierry Gallouët, Cindy Guichard, Raphaele Herbin. An error estimate for the approximation of linear parabolic equations by the Gradient Discretization Method. FVCA 2017 - International Conference on Finite Volumes for Complex Applications VIII, 2017, Lille, France. hal-01442921

\author{
HAL Id: hal-01442921 \\ https://hal.science/hal-01442921
}

Submitted on 21 Jan 2017

HAL is a multi-disciplinary open access archive for the deposit and dissemination of scientific research documents, whether they are published or not. The documents may come from teaching and research institutions in France or abroad, or from public or private research centers.
L'archive ouverte pluridisciplinaire HAL, est destinée au dépôt et à la diffusion de documents scientifiques de niveau recherche, publiés ou non, émanant des établissements d'enseignement et de recherche français ou étrangers, des laboratoires publics ou privés. 


\title{
An error estimate for the approximation of linear parabolic equations by the Gradient Discretization Method
}

\author{
J. Droniou, R. Eymard, T. Gallouët, C. Guichard and R. Herbin
}

\begin{abstract}
We establish an error estimate for fully discrete time-space gradient schemes on a simple linear parabolic equation. This error estimate holds for all the schemes within the framework of the gradient discretisation method: conforming and non conforming finite element, mixed finite element, hybrid mixed mimetic family, some Multi-Point Flux approximation finite volume scheme and some discontinuous Galerkin schemes.
\end{abstract}

Key words: Heat equation, error estimate, gradient discretisation method

MSC (2010): 65M60,65M12

Jérôme Droniou

School of Mathematical Sciences, Monash University, Victoria 3800, Australia, e-mail: jerome.droniou@monash.edu

Robert Eymard

Laboratoire d'Analyse et de Mathématiques Appliquées, CNRS, UPEM, UPEC, 5 boulevard Descartes, Champs-sur-Marne 77454 Marne-la-Vallée Cedex 2, France. e-mail: Robert.Eymard@u-pem.fr

T. Gallouët

Aix Marseille Univ, CNRS, Centrale Marseille, I2M, Marseille, France, e-mail: Thierry.Gallouet@univ-amu.fr

C. Guichard

ANGE project-team, Laboratoire Jacques-Louis Lions (LJLL), UMR 7598 CNRS, Sorbonne Universités, UPMC Univ. Paris 6, and INRIA de Paris, and CEREMA, France e-mail: guichard@1jll.math.upmc.fr

R. Herbin

Aix Marseille Univ, CNRS, Centrale Marseille, I2M, Marseille, France, e-mail: Raphaele.Herbin@univ-amu.fr. 


\section{Introduction}

The Gradient Discretization method (GDM) [5, 3] provides a common mathematical framework for a number of numerical schemes dedicated to the approximation of elliptic or parabolic problems, linear or nonlinear, coupled or not; these include conforming and non conforming finite element, mixed finite element, hybrid mixed mimetic schemes [4] and some Multi-Point Flux Approximation [1] and Discrete Duality finite volume schemes [2] : we refer to [3, Part III] for more on this (note that in the present proceedings, it is shown that in some way the Discontinuous Galerkin schemes may also enter this framework [6]). Let us recall this framework in the case of the following linear elliptic problem:

$$
\left\{\begin{array}{l}
\text { Find } \bar{u} \in H_{0}^{1}(\Omega) \text { such that, for all } v \in H_{0}^{1}(\Omega), \\
\int_{\Omega} \nabla \bar{u}(\boldsymbol{x}) \cdot \nabla v(\boldsymbol{x}) \mathrm{d} \boldsymbol{x}=\int_{\Omega} f(\boldsymbol{x}) v(\boldsymbol{x}) \mathrm{d} \boldsymbol{x}
\end{array}\right.
$$

Any numerical scheme that fits into the GDM is defined in the following way. A finite dimensional real vector space $X_{\mathscr{D}, 0}$ is chosen, which describes the discrete unknowns, along with two linear operators $\Pi_{\mathscr{D}}: X_{\mathscr{D}, 0} \rightarrow L^{2}(\Omega)$ and $\nabla_{\mathscr{D}}: X_{\mathscr{D}, 0} \rightarrow$ $L^{2}(\Omega)^{d}$, which respectively reconstruct, from the discrete unknowns, a function on $\Omega$, and its "gradient", specified in such a way that $\|\cdot\|_{L^{2}(\Omega)^{d}}$ is a norm on $X_{\mathscr{D}, 0}$. Then, the scheme is written by replacing in (1) the continuous space and operators by their discrete equivalent:

$$
\left\{\begin{array}{l}
\text { Find } u_{\mathscr{D}} \in X_{\mathscr{D}, 0} \text { such that, for all } v_{\mathscr{D}} \in X_{\mathscr{D}, 0}, \\
\int_{\Omega} \nabla_{\mathscr{D}} u_{\mathscr{D}}(\boldsymbol{x}) \cdot \nabla_{\mathscr{D}} v_{\mathscr{D}}(\boldsymbol{x}) \mathrm{d} \boldsymbol{x}=\int_{\Omega} f(\boldsymbol{x}) \Pi_{\mathscr{D}} v_{\mathscr{D}}(\boldsymbol{x}) \mathrm{d} \boldsymbol{x} .
\end{array}\right.
$$

Of course, there are as many schemes as there are choices of $\left(X_{\mathscr{D}, 0}, \Pi_{\mathscr{D}}, \nabla_{\mathscr{D}}\right)$, which is the reason why the GDM contains many different numerical methods. We then define

$$
\begin{aligned}
& C_{\mathscr{D}}=\max _{v \in X_{\mathscr{D}, 0} \backslash\{0\}} \frac{\left\|\Pi_{\mathscr{D}} v\right\|_{L^{2}}}{\left\|\nabla_{\mathscr{D}} v\right\|_{L^{2}}}, \\
& \forall \boldsymbol{\varphi} \in H_{0}^{1}(\Omega), S_{\mathscr{D}}(\boldsymbol{\varphi})=\min _{v \in X_{\mathscr{D}, 0}}\left(\left\|\Pi_{\mathscr{D}} v-\varphi\right\|_{L^{2}}^{2}+\left\|\nabla_{\mathscr{D}} v-\nabla \varphi\right\|_{L^{2}}^{2}\right)^{1 / 2}, \\
& \forall \boldsymbol{\varphi} \in H_{\operatorname{div}}(\Omega), \\
& \quad W_{\mathscr{D}}(\boldsymbol{\varphi})=\max _{v \in X_{\mathscr{D}, 0} \backslash\{0\}} \frac{1}{\left\|\nabla_{\mathscr{D}} v\right\|_{L^{2}}}\left|\int_{\Omega}\left(\nabla_{\mathscr{D}} v(\boldsymbol{x}) \cdot \boldsymbol{\varphi}(\boldsymbol{x})+\Pi_{\mathscr{D}} v(\boldsymbol{x}) \operatorname{div} \boldsymbol{\varphi}(\boldsymbol{x})\right) \mathrm{d} \boldsymbol{x}\right|
\end{aligned}
$$

Following the proof in [3], it can be established that, if $\bar{u}$ is the unique solution to (1) and $u_{\mathscr{D}}$ is the unique solution to (2), the following error estimates hold

$$
\begin{aligned}
& \left\|\nabla \bar{u}-\nabla_{\mathscr{D}} u_{\mathscr{D}}\right\|_{L^{2}(\Omega)^{d}} \leq W_{\mathscr{D}}(\nabla \bar{u})+S_{\mathscr{D}}(\bar{u}), \\
& \left\|\bar{u}-\Pi_{\mathscr{D}} u_{\mathscr{D}}\right\|_{L^{2}(\Omega)} \leq C_{\mathscr{D}} W_{\mathscr{D}}(\nabla \bar{u})+\left(C_{\mathscr{D}}+1\right) S_{\mathscr{D}}(\bar{u}) .
\end{aligned}
$$


Remark 1. In [3], the definition of $S_{\mathscr{D}}$ is slightly different from (4). This has no consequence on the proof of (6)-(7).

It can then be proved [3] that on all the mesh-based schemes which enter the Gradient Discretization Method, there exists $C \geq 0$, only depending on regularity factors of the mesh, such that

$$
\begin{aligned}
& \forall \varphi \in W^{2, \infty}(\Omega) \cap H_{0}^{1}(\Omega), S_{\mathscr{D}}(\varphi) \leq C h_{\mathscr{M}}\|\varphi\|_{W^{2, \infty}(\Omega)}, \\
& \forall \boldsymbol{\varphi} \in W^{1, \infty}(\Omega)^{d}, W_{\mathscr{D}}(\boldsymbol{\varphi}) \leq C h_{\mathscr{M}}\|\boldsymbol{\varphi}\|_{W^{1, \infty}(\Omega)^{d}}
\end{aligned}
$$

where the mesh size $h_{\mathscr{M}}$ is the maximum diameter of all the grid cells. For these methods, a linear error estimate with respect to the mesh size may be derived from (6)-(7) in the case of sufficiently regular solutions.

The aim of this paper is to establish an error estimate for the GDM applied to linear parabolic problems; error estimates for such problems are classical in the framework of finite differences, finite elements and finite volumes. The advantage of the GDM framework is that this error estimate applies to all the above mentioned schemes. We consider here the GDM with an implicit Euler time-stepping for the approximation of the heat equation with homogeneous Dirichlet boundary conditions, which reads:

$$
\begin{aligned}
\partial_{t} \bar{u}-\Delta \bar{u} & =f \text { in } \Omega \times(0, T), \\
\bar{u}(\cdot, 0) & =u_{\text {ini }} \text { on } \Omega, \\
\bar{u} & =0 \text { on } \partial \Omega \times(0, T) .
\end{aligned}
$$

The diffusion operator $-\Delta u$ could easily be replaced by $-\operatorname{div}(\Lambda \nabla u)$, with a uniformly coercive and bounded $\Lambda$, without major change in the analysis. The following hypotheses are assumed throughout this paper:

$$
\begin{aligned}
& \Omega \text { is an open bounded connected subset of } \mathbb{R}^{d}\left(d \in \mathbb{N}^{\star}\right) \text { and } T>0, \\
& f \in L^{2}(\Omega \times(0, T)), \\
& u_{\text {ini }} \in L^{2}(\Omega) .
\end{aligned}
$$

Under these assumptions, there exists a unique function $\bar{u}$ of (9) in the following (weak) sense:

$$
\left\{\begin{array}{l}
\bar{u} \in L^{2}\left(0, T ; H_{0}^{1}(\Omega)\right) \cap C\left([0, T] ; L^{2}(\Omega)\right), \partial_{t} \bar{u} \in L^{2}\left(0, T ; H^{-1}(\Omega)\right) \\
\bar{u}(\cdot, 0)=u_{\text {ini }} \text { and, for all } w \in L^{2}\left(0, T ; H_{0}^{1}(\Omega)\right) \\
\int_{0}^{T}\left\langle\partial_{t} \bar{u}(\cdot, t), w(\cdot, t)\right\rangle_{H^{-1}, H_{0}^{1}} \mathrm{~d} t \\
+\int_{0}^{T} \int_{\Omega} \nabla \bar{u}(\boldsymbol{x}, t) \cdot \nabla w(\boldsymbol{x}, t) \mathrm{d} \boldsymbol{x} \mathrm{d} t=\int_{0}^{T} \int_{\Omega} f(\boldsymbol{x}, t) w(\boldsymbol{x}, t) \mathrm{d} \boldsymbol{x} \mathrm{d} t
\end{array}\right.
$$

This paper is organized as follows. In Section 2, we define the GDM for the approximation of space-time parabolic problems, and we define, inspired by (8), the notion of space size of a gradient discretization. We then apply in Section 3 the 
GDM to obtain a generic numerical scheme ("gradient scheme") for the approximation of Problem (11). We then prove Theorem 1, which states an error estimate under regularity hypotheses on the solution $\bar{u}$ of Problem (11). Throughout this paper, $\|\cdot\|_{L^{2}(\Omega)}$ and $\|\cdot\|_{L^{2}(\Omega)^{d}}$ are abridged as $\|\cdot\|_{L^{2}}$.

\section{Space-time Gradient Discretization}

Definition 1 (Space-time Gradient Discretization). A space-time gradient discretisation is $\mathscr{D}_{T}=\left(X_{\mathscr{D}, 0}, \Pi_{\mathscr{D}}, \nabla_{\mathscr{D}}, \mathscr{I}_{\mathscr{D}},\left(t^{(n)}\right)_{n=0, \ldots, N}\right)$ with

- $X_{\mathscr{D}, 0}$ a finite dimensional real vector space describing the discrete unknowns,

- $\Pi_{\mathscr{D}}: X_{\mathscr{D}, 0} \rightarrow L^{2}(\Omega)$ a linear mapping reconstructing a function defined over $\Omega$ from an element of $X_{\mathscr{D}, 0}$,

- $\nabla_{\mathscr{D}}: X_{\mathscr{D}, 0} \rightarrow L^{2}(\Omega)^{d}$ a linear mapping which reconstructs, from an element of $X_{\mathscr{D}, 0}$, a "gradient" (vector-valued function) over $\Omega$. This gradient reconstruction must be chosen such that $\left\|\nabla_{\mathscr{D}} \cdot\right\|_{L^{2}}$ is a norm on $X_{\mathscr{D}, 0}$.

- $\mathscr{I}_{\mathscr{D}}: L^{2}(\Omega) \rightarrow X_{\mathscr{D}, 0}$ is a linear and continuous interpolation operator for the initial conditions,

- $t^{(0)}=0<t^{(1)} \ldots<t^{(N)}=T$.

We set $\delta t^{\left(n+\frac{1}{2}\right)}=t^{(n+1)}-t^{(n)}$, for $n=0, \ldots, N-1$, and $\delta t_{\mathscr{D}}=\max _{n=0, \ldots, N-1} \delta t^{\left(n+\frac{1}{2}\right)}$.

To a family $v=\left(v^{(n)}\right)_{n=0, \ldots, N} \in X_{\mathscr{D}, 0}^{N+1}$ we associate the functions $v_{\text {imp }} \in L^{\infty}\left(0, T ; X_{\mathscr{D}, 0}\right)$, $\Pi_{\mathscr{D}} v \in L^{\infty}\left(0, T ; L^{2}(\Omega)\right)$, and $\nabla_{\mathscr{D} v} \in L^{\infty}\left(0, T ; L^{2}(\Omega)^{d}\right)$ defined by

$$
\begin{aligned}
& \forall n=0, \ldots, N-1, \forall t \in\left(t^{(n)}, t^{(n+1)}\right], v_{\text {imp }}(t)=v^{(n+1)} \text { and, } \\
& \text { for a.e. } \boldsymbol{x} \in \Omega, \Pi_{\mathscr{D} v}(\boldsymbol{x}, t)=\Pi_{\mathscr{D}}\left[v_{\mathrm{imp}}(t)\right](\boldsymbol{x}), \nabla_{\mathscr{D}} v(\boldsymbol{x}, t)=\nabla_{\mathscr{D}}\left[v_{\mathrm{imp}}(t)\right](\boldsymbol{x}) .
\end{aligned}
$$

Define also, for $v \in X_{\mathscr{D}, 0}^{N+1}$,

$$
\forall n=0, \ldots, N-1, \delta_{\mathscr{D}}^{\left(n+\frac{1}{2}\right)} v:=\frac{\Pi_{\mathscr{D}} v^{(n+1)}-\Pi_{\mathscr{D}} v^{(n)}}{\delta t^{\left(n+\frac{1}{2}\right)}}
$$

In the following, $L^{2}(\Omega) \times L^{2}(\Omega)^{d}$ is endowed with the Hilbertian norm defined by $\|(\varphi, \boldsymbol{\psi})\|^{2}=\|\varphi\|_{L^{2}}^{2}+\|\boldsymbol{\psi}\|_{L^{2}}^{2}$.

Lemma 1 (Linear spatial interpolator). Under Hypotheses (10), let $\mathscr{D}_{T}$ be a space-time GD in the sense of Definition 1. Then the relation

$$
\forall \varphi \in H_{0}^{1}(\Omega), P_{\mathscr{D}} \varphi \in \underset{w \in X_{\mathscr{D}, 0}}{\operatorname{argmin}}\left(\left\|\Pi_{\mathscr{D}} w-\varphi\right\|_{L^{2}}^{2}+\left\|\nabla_{\mathscr{D} w}-\nabla \varphi\right\|_{L^{2}}^{2}\right),
$$

defines a unique linear continuous operator $P_{\mathscr{D}}: H_{0}^{1}(\Omega) \rightarrow X_{\mathscr{D}, 0}$, which statisfies

$$
\forall \varphi \in H_{0}^{1}(\Omega), S_{\mathscr{D}}(\varphi)=\left(\left\|\Pi_{\mathscr{D}} P_{\mathscr{D}} \varphi-\varphi\right\|_{L^{2}}^{2}+\left\|\nabla_{\mathscr{D}} P_{\mathscr{D}} \varphi-\nabla \varphi\right\|_{L^{2}}^{2}\right)^{1 / 2} .
$$


Proof. Set $V=\left\{\left(\Pi_{\mathscr{D} w}, \nabla_{\mathscr{D}} w\right): w \in X_{\mathscr{D}, 0}\right\}$ and let $\mathscr{P}: L^{2}(\Omega) \times L^{2}(\Omega)^{d} \rightarrow V$ be the orthogonal projection. Since $\left\|\nabla_{\mathscr{D}}\right\|_{L^{2}}$ is a norm on $X_{\mathscr{D}, 0}$, for any $z \in V$ there exists a unique $\mathscr{R} z \in X_{\mathscr{D}, 0}$ such that $\left(\Pi_{\mathscr{D}} \mathscr{R} z, \nabla_{\mathscr{D}} \mathscr{R} z\right)=z$. This defines a linear continuous mapping $\mathscr{R}: V \rightarrow X_{\mathscr{D}, 0}$, and (14) shows that $P_{\mathscr{D}} \varphi=\mathscr{R} \circ \mathscr{P}(\varphi, \nabla \varphi)$. Hence, $P_{\mathscr{D}} \varphi$ is uniquely defined and $P_{\mathscr{D}}$ is linear continuous. Relation (15) follows from Definition (4) of $S_{\mathscr{D}}$.

Using this operator $P_{\mathscr{D}}$, we can introduce the following definition.

Definition 2 (Space size of a space-time gradient discretization). Under Hypotheses (10), let $\mathscr{D}_{T}$ be a space-time GD in the sense of Definition 1. Define the following norms of continuous linear or bilinear operators:

$$
\begin{gathered}
\left\|\mathbb{S}_{\mathscr{D}}\right\|=\sup \left\{\frac{\left\|\mathbb{S}_{\mathscr{D}}(\varphi)\right\|_{L^{2} \times L^{2}}}{\|\varphi\|_{W^{2, \infty}(\Omega)}}: \varphi \in W^{2, \infty}(\Omega) \cap H_{0}^{1}(\Omega) \backslash\{0\}\right\}, \\
\text { where } \mathbb{S}_{\mathscr{D}}(\boldsymbol{\varphi})=\left(\varphi-\Pi_{\mathscr{D}} P_{\mathscr{D}} \varphi, \nabla \varphi-\nabla_{\mathscr{D}} P_{\mathscr{D}} \varphi\right), \\
\left\|\mathbb{W}_{\mathscr{D}}\right\|=\sup \left\{\frac{\left|\mathbb{W}_{\mathscr{D}}(\boldsymbol{\varphi}, v)\right|}{\|\boldsymbol{\varphi}\|_{W^{1, \infty}(\Omega)^{d}}\left\|\nabla_{\mathscr{D}}\right\|_{L^{2}}}:(\boldsymbol{\varphi}, v) \in\left(W^{1, \infty}(\Omega)^{d}\right) \backslash\{0\} \times X_{\mathscr{D}, 0} \backslash\{0\}\right\}, \\
\text { where } \mathbb{W}_{\mathscr{D}}(\boldsymbol{\varphi}, v)=\int_{\Omega}\left(\operatorname{div} \boldsymbol{\varphi}(\boldsymbol{x}) \Pi_{\mathscr{D}} v(\boldsymbol{x})+\boldsymbol{\varphi}(\boldsymbol{x}) \cdot \nabla_{\mathscr{D}} v(\boldsymbol{x})\right) \mathrm{d} \boldsymbol{x},
\end{gathered}
$$

and

$$
\left\|\mathbb{I}_{\mathscr{D}}\right\|=\sup \left\{\frac{\left\|\mathbb{I}_{\mathscr{D}}(\varphi)\right\|_{L^{2}}}{\|\varphi\|_{W^{1, \infty}(\Omega)}}: \varphi \in W^{1, \infty}(\Omega) \backslash\{0\}\right\}, \text { where } \mathbb{I}_{\mathscr{D}}(\varphi)=\varphi-\Pi_{\mathscr{D}} \mathscr{I}_{\mathscr{D}} \varphi
$$

The space size of the space-time GD is then defined by

$$
h_{\mathscr{D}}=\max \left(\left\|\mathbb{S}_{\mathscr{D}}\right\|,\left\|\mathbb{W}_{\mathscr{D}}\right\|,\left\|\mathbb{I}_{\mathscr{D}}\right\|\right) .
$$

It therefore satisfies

$$
\begin{aligned}
& \forall \varphi \in W^{2, \infty}(\Omega) \cap H_{0}^{1}(\Omega), S_{\mathscr{D}}(\varphi) \leq h_{\mathscr{D}}\|\varphi\|_{W^{2, \infty}(\Omega)}, \\
& \forall \boldsymbol{\varphi} \in W^{1, \infty}(\Omega)^{d}, W_{\mathscr{D}}(\boldsymbol{\varphi}) \leq h_{\mathscr{D}}\|\boldsymbol{\varphi}\|_{W^{1, \infty}(\Omega)^{d}}, \\
& \forall \varphi \in W^{1, \infty}(\Omega),\left\|\Pi_{\mathscr{D}} \mathscr{I}_{\mathscr{D}} \varphi-\varphi\right\|_{L^{2}} \leq h_{\mathscr{D}}\|\varphi\|_{W^{1, \infty}(\Omega)} .
\end{aligned}
$$

Remark 2 (Link between $h_{\mathscr{D}}$ and the size of the mesh for mesh-based gradient discretizations). If the gradient discretization is based on a polytopal mesh (see [3, Definition 7.2]), and if $\mathscr{I}_{\mathscr{D}}$ is defined by means of local average values around the degrees of freedom, then, for all the mesh-based examples of gradient discretizations given in [3], there exists $C>0$ only depending on regularity factors of the mesh such that $h_{\mathscr{D}} \leq C h_{\mathscr{M}}$. 


\section{Gradient Scheme and error estimate}

Using an implicit scheme for the time stepping, the GDM applied to Problem (11) leads to the following gradient scheme: find a family $\left(u^{(n)}\right)_{n=0, \ldots, N} \in X_{\mathscr{D}, 0}^{N+1}$ such that

$$
\left\{\begin{array}{l}
u^{(0)}=\mathscr{I}_{\mathscr{D}} u_{\text {ini }} \text { and, for all } n=0, \ldots, N-1, u^{(n+1)} \text { satisfies: } \forall v \in X_{\mathscr{D}, 0} \\
\int_{\Omega} \delta_{\mathscr{D}}^{\left(n+\frac{1}{2}\right)} u(\boldsymbol{x}) \Pi_{\mathscr{D}} v(\boldsymbol{x}) \mathrm{d} \boldsymbol{x}+\int_{\Omega} \nabla_{\mathscr{D}} u^{(n+1)}(\boldsymbol{x}) \cdot \nabla_{\mathscr{D}} v(\boldsymbol{x}) \mathrm{d} \boldsymbol{x} \\
=\frac{1}{\delta t^{\left(n+\frac{1}{2}\right)}} \int_{t^{(n)}}^{t^{(n+1)}} \int_{\Omega} f(\boldsymbol{x}, t) \Pi_{\mathscr{D}} v(\boldsymbol{x}) \mathrm{d} \boldsymbol{x} \mathrm{d} t
\end{array}\right.
$$

Here, of course, $u^{(n)}$ is expected to provide an approximation of $\bar{u}$ at time $t_{n}$. Let us now state our main result.

Theorem 1 (Error estimate, linear case and regular solution). Under Hypotheses $(10)$, let $\mathscr{D}_{T}$ be a space-time $G D$ in the sense of Definition 1. Let $h_{\mathscr{D}}$ be defined by Definition 2. Assume that the solution $\bar{u}$ to (11) belongs to $W^{1, \infty}\left(0, T ; W^{2, \infty}(\Omega)\right)$, and let $u$ be the solution to the implicit $G S(18)$. Then there exists $C>0$, depending only on $\bar{u}, \Omega, T$ and (in a non-decreasing way) of $C_{\mathscr{D}}$ (defined by (3)), such that

$$
\max _{t \in[0, T]}\left\|\Pi_{\mathscr{D}} u(\cdot, t)-\bar{u}(\cdot, t)\right\|_{L^{2}}+\left\|\nabla_{\mathscr{D}} u-\nabla \bar{u}\right\|_{L^{2}(\Omega \times(0, T))^{d}} \leq C\left(\delta t_{\mathscr{D}}+h_{\mathscr{D}}\right) .
$$

Proof of Theorem 1. Here, $C_{i}$ denote various quantities having the same dependencies as $C$ in the theorem. Let $\bar{u}^{(0)}=\bar{u}(0)$ and, if $n \in\{0, \ldots, N-1\}$ and $g=f, \bar{u}$ or $\partial_{t} \bar{u}$,

$$
g^{(n+1)}(\boldsymbol{x})=\frac{1}{\delta t^{\left(n+\frac{1}{2}\right)}} \int_{t^{(n)}}^{t^{(n+1)}} g(\boldsymbol{x}, t) \mathrm{d} t .
$$

Step 1: Preliminary estimates. Since $\bar{u} \in C\left([0, T] ; W^{2, \infty}(\Omega)\right)$, we can write (15) with $\varphi=\bar{u}\left(t^{(n+1)}\right)$ and use (17a). The regularity on $\bar{u}$ also ensures that $\nabla \bar{u}:[0, T] \rightarrow$ $L^{2}(\Omega)^{d}$ is Lipschitz-continuous. Thus, recalling the definition (19) of $\bar{u}^{(n+1)}$,

$$
\begin{aligned}
\| \nabla \bar{u}^{(n+1)} & -\nabla_{\mathscr{D}} P_{\mathscr{D}} \bar{u}\left(t^{(n+1)}\right) \|_{L^{2}} \\
& \leq\left\|\nabla \bar{u}^{(n+1)}-\nabla \bar{u}\left(t^{(n+1)}\right)\right\|_{L^{2}}+S_{\mathscr{D}}\left(\bar{u}\left(t^{(n+1)}\right)\right) \leq C_{1}\left(\delta t_{\mathscr{D}}+h_{\mathscr{D}}\right) .
\end{aligned}
$$

Since $\partial_{t} \bar{u} \in L^{\infty}\left(0, T ; W^{2, \infty}(\Omega)\right),\left(\left\|\partial_{t} \bar{u}^{(n+1)}\right\|_{W^{2, \infty}(\Omega)}\right)_{n \in \mathbb{N}}$ is bounded. Applying (15) to $\varphi=\partial_{t} \bar{u}^{(n+1)}=\left[\bar{u}\left(t^{(n+1)}\right)-\bar{u}\left(t^{(n)}\right)\right] / \delta t^{\left(n+\frac{1}{2}\right)}$, using the linearity of $P_{\mathscr{D}}$ and invoking (17a), we obtain

$$
\left\|\frac{\Pi_{\mathscr{D}} P_{\mathscr{D}} \bar{u}\left(t^{(n+1)}\right)-\Pi_{\mathscr{D}} P_{\mathscr{D}} \bar{u}\left(t^{(n)}\right)}{\delta t^{\left(n+\frac{1}{2}\right)}}-\partial_{t} \bar{u}^{(n+1)}\right\|_{L^{2}} \leq C_{2} h_{\mathscr{D}} .
$$


Step 2: Proof of the error estimate. Since $\nabla \bar{u} \in L^{\infty}\left(0, T ; W^{1, \infty}(\Omega)^{d}\right),\left(\nabla \bar{u}^{(n+1)}\right)_{n \in \mathbb{N}}$ is bounded in $W^{1, \infty}(\Omega)^{d}$. Hence, using the definition of $W_{\mathscr{D}}$ and $(17 \mathrm{~b})$, for all $v \in X_{\mathscr{D}, 0}, \int_{\Omega}\left(\Pi_{\mathscr{D}} v(\boldsymbol{x}) \operatorname{div}\left(\nabla \bar{u}^{(n+1)}\right)(\boldsymbol{x})+\nabla \bar{u}^{(n+1)}(\boldsymbol{x}) \cdot \nabla_{\mathscr{D}} v(\boldsymbol{x})\right) \mathrm{d} \boldsymbol{x} \leq C_{3} h_{\mathscr{D}}\left\|\nabla_{\mathscr{D}} v\right\|_{L^{2}}$. Owing to the regularity of $\bar{u}$, the equation $\partial_{t} \bar{u}-f=\operatorname{div}(\nabla \bar{u})$ is satisfied a.e. in space and time. Averaging over time in $\left(t^{(n)}, t^{(n+1)}\right)$ gives $\partial_{t} \bar{u}^{(n+1)}-f^{(n+1)}=\operatorname{div}\left(\nabla \bar{u}^{(n+1)}\right)$ a.e. in space, and thus

$\int_{\Omega}\left(\Pi_{\mathscr{D}} v(\boldsymbol{x})\left(\partial_{t} \bar{u}^{(n+1)}(\boldsymbol{x})-f^{(n+1)}(\boldsymbol{x})\right)+\nabla \bar{u}^{(n+1)}(\boldsymbol{x}) \cdot \nabla_{\mathscr{D}} v(\boldsymbol{x})\right) \mathrm{d} \boldsymbol{x} \leq C_{3} h_{\mathscr{D}}\left\|\nabla_{\mathscr{D}} v\right\|_{L^{2}}$.

Use the GS (18) to replace the term $f^{(n+1)}$ in the left-hand side:

$$
\begin{aligned}
\int_{\Omega} \Pi_{\mathscr{D}} v & (\boldsymbol{x})\left(\partial_{t} \bar{u}^{(n+1)}(\boldsymbol{x})-\delta_{\mathscr{D}}^{\left(n+\frac{1}{2}\right)} u(\boldsymbol{x})\right) \mathrm{d} \boldsymbol{x} \\
& \quad+\int_{\Omega}\left(\nabla \bar{u}^{(n+1)}(\boldsymbol{x})-\nabla_{\mathscr{D}} u^{(n+1)}(\boldsymbol{x})\right) \cdot \nabla_{\mathscr{D}} v(\boldsymbol{x}) \mathrm{d} \boldsymbol{x} \leq C_{3} h_{\mathscr{D}}\left\|\nabla_{\mathscr{D}} v\right\|_{L^{2}}
\end{aligned}
$$

For $k=0, \ldots, N$, set $e^{(k)}=P_{\mathscr{D}} \bar{u}\left(t^{(k)}\right)-u^{(k)}$ and notice that

$$
\delta_{\mathscr{D}}^{\left(n+\frac{1}{2}\right)} e=\left[\frac{\Pi_{\mathscr{D}} P_{\mathscr{D}} \bar{u}\left(t^{(n+1)}\right)-\Pi_{\mathscr{D}} P_{\mathscr{D}} \bar{u}\left(t^{(n)}\right)}{\delta t^{\left(n+\frac{1}{2}\right)}}-\partial_{t} \bar{u}^{(n+1)}\right]+\left[\partial_{t} \bar{u}^{(n+1)}-\delta_{\mathscr{D}}^{\left(n+\frac{1}{2}\right)} u\right],
$$

and $\nabla_{\mathscr{D}} e^{(n+1)}=\left[\nabla_{\mathscr{D}} P_{\mathscr{D}} \bar{u}\left(t^{(n+1)}\right)-\nabla \bar{u}^{(n+1)}\right]+\left[\nabla \bar{u}^{(n+1)}-\nabla_{\mathscr{D}} u^{(n+1)}\right]$.

Then (22), (21), (20) and the definition of $C_{\mathscr{D}}$ give

$$
\int_{\Omega} \Pi_{\mathscr{D}} v(\boldsymbol{x}) \delta_{\mathscr{D}}^{\left(n+\frac{1}{2}\right)} e(\boldsymbol{x}) \mathrm{d} \boldsymbol{x}+\int_{\Omega} \nabla_{\mathscr{D}} e^{(n+1)}(\boldsymbol{x}) \cdot \nabla_{\mathscr{D}} v(\boldsymbol{x}) \mathrm{d} \boldsymbol{x} \leq C_{4}\left(\delta t_{\mathscr{D}}+h_{\mathscr{D}}\right)\left\|\nabla_{\mathscr{D}} v\right\|_{L^{2}}
$$

Take $v=\delta t^{\left(n+\frac{1}{2}\right)} e^{(n+1)}$ and sum over $n=0, \ldots, m-1$ for some $m \in\{1, \ldots, N\}$ :

$$
\begin{gathered}
\sum_{n=0}^{m-1} \int_{\Omega} \Pi_{\mathscr{D}} e^{(n+1)}(\boldsymbol{x})\left[\Pi_{\mathscr{D}} e^{(n+1)}(\boldsymbol{x})-\Pi_{\mathscr{D}} e^{(n)}(\boldsymbol{x})\right] \mathrm{d} \boldsymbol{x}+\sum_{n=0}^{m-1} \delta t^{\left(n+\frac{1}{2}\right)}\left\|\nabla_{\mathscr{D}} e^{(n+1)}\right\|_{L^{2}}^{2} \\
\leq \sum_{n=0}^{m-1} C_{4}\left(\delta t_{\mathscr{D}}+h_{\mathscr{D}}\right) \delta t^{\left(n+\frac{1}{2}\right)}\left\|\nabla_{\mathscr{D}} e^{(n+1)}\right\|_{L^{2}}
\end{gathered}
$$

Apply the relation $b(b-a)=\frac{1}{2} b^{2}-\frac{1}{2} a^{2}+\frac{1}{2}(b-a)^{2} \geq \frac{1}{2} b^{2}-\frac{1}{2} a^{2}$ to $a=\Pi_{\mathscr{D}} e^{(n)}(\boldsymbol{x})$ and $b=\Pi_{\mathscr{D}} e^{(n+1)}(\boldsymbol{x})$. The Young inequality in the right-hand side of (23) leads to

$$
\begin{array}{r}
\frac{1}{2} \int_{\Omega}\left(\Pi_{\mathscr{D}} e^{(m)}(\boldsymbol{x})\right)^{2} \mathrm{~d} \boldsymbol{x}+\sum_{n=0}^{m-1} \delta t^{\left(n+\frac{1}{2}\right)}\left\|\nabla_{\mathscr{D}} e^{(n+1)}\right\|_{L^{2}}^{2} \leq \frac{1}{2} \int_{\Omega}\left(\Pi_{\mathscr{D}} e^{(0)}(\boldsymbol{x})\right)^{2} \mathrm{~d} \boldsymbol{x} \\
+\frac{1}{2} \sum_{n=0}^{m-1} \delta t^{\left(n+\frac{1}{2}\right)}\left\|\nabla_{\mathscr{D}} e^{(n+1)}\right\|_{L^{2}}^{2}+\frac{1}{2} \sum_{n=0}^{m-1} C_{4}^{2}\left(\delta t_{\mathscr{D}}+h_{\mathscr{D}}\right)^{2} \delta t^{\left(n+\frac{1}{2}\right)}
\end{array}
$$


Owing to (17c) and Estimate (15), since $u^{(0)}=\mathscr{I}_{\mathscr{D}} u_{\text {ini }}=\mathscr{I}_{\mathscr{D}} \bar{u}(0)$,

$$
\left\|\Pi_{\mathscr{D}} e^{(0)}\right\|_{L^{2}} \leq\left\|\Pi_{\mathscr{D}} P_{\mathscr{D}} \bar{u}(0)-\bar{u}(0)\right\|_{L^{2}}+\left\|\bar{u}(0)-\Pi_{\mathscr{D}} \mathscr{I}_{\mathscr{D}} \bar{u}(0)\right\|_{L^{2}} \leq C_{5} h_{\mathscr{D}}
$$

Recalling the definition of the space-time function $\nabla_{\mathscr{D}} e$ and using $\sum_{n=0}^{m-1} \delta t^{\left(n+\frac{1}{2}\right)} \leq$ $T$, Equation (24) yields

$$
\frac{1}{2}\left\|\Pi_{\mathscr{D}} e^{(m)}\right\|_{L^{2}}^{2}+\frac{1}{2} \sum_{n=0}^{m-1} \delta t^{\left(n+\frac{1}{2}\right)}\left\|\nabla_{\mathscr{D}} e^{(n+1)}\right\|_{L^{2}}^{2} \leq C_{6}\left(\delta t_{\mathscr{D}}+h_{\mathscr{D}}\right)^{2}
$$

The definition of $e^{(m)}$, a triangle inequality, (15) and Equation (25) lead on one hand to the following estimate, valid for all $m=1, \ldots, N-1$ :

$$
\left\|\Pi_{\mathscr{D}} u^{(m)}-\bar{u}\left(t^{(m)}\right)\right\|_{L^{2}} \leq C_{7}\left(\delta t_{\mathscr{D}}+h_{\mathscr{D}}\right)+\sqrt{2} S_{\mathscr{D}}\left(\bar{u}\left(t^{(m)}\right)\right) \leq C_{8}\left(\delta t_{\mathscr{D}}+h_{\mathscr{D}}\right) .
$$

On the other hand, using again (15) and a triangle inequality, Equation (25) with $m=N-1$ leads to

$$
\begin{aligned}
& \sum_{n=0}^{N-1} \delta t^{\left(n+\frac{1}{2}\right)}\left\|\nabla_{\mathscr{D}} u^{(n+1)}-\nabla \bar{u}\left(t^{(n+1)}\right)\right\|_{L^{2}}^{2} \\
& \leq 4 C_{6}\left(\delta t_{\mathscr{D}}+h_{\mathscr{D}}\right)^{2}+4 \sum_{n=0}^{N-1} \delta t^{\left(n+\frac{1}{2}\right)} S_{\mathscr{D}}\left(\bar{u}\left(t^{(n+1)}\right)\right)^{2} \leq C_{9}^{2}\left(\delta t_{\mathscr{D}}+h_{\mathscr{D}}\right)^{2}
\end{aligned}
$$

The conclusion follows from (26), (27) and the Lipschitz-continuity of $\bar{u}:[0, T] \rightarrow$ $H^{1}(\Omega)$ to compare $\bar{u}(t)($ resp. $\nabla \bar{u}(t))$ with $\bar{u}\left(t^{(n+1)}\right)$ (resp. $\left.\nabla \bar{u}\left(t^{(n+1)}\right)\right)$ when $t \in$ $\left(t^{(n)}, t^{(n+1)}\right]$.

\section{References}

1. Aavatsmark, I., Barkve, T., Boe, O., Mannseth, T.: Discretization on non-orthogonal, quadrilateral grids for inhomogeneous, anisotropic media. J. Comput. Phys. 127(1), 2-14 (1996). DOI http://dx.doi.org/10.1006/jcph.1996.0154

2. Coudière, Y., Hubert, F.: A 3d discrete duality finite volume method for nonlinear elliptic equations. SIAM Journal on Scientific Computing 33(4), 1739-1764 (2011)

3. Droniou, J., Eymard, R., Gallouët, T., Guichard, C., Herbin, R.: The gradient discretisation method: A framework for the discretisation and numerical analysis of linear and nonlinear elliptic and parabolic problems (2016). URL https://hal.archives-ouvertes.fr/hal-01382358

4. Droniou, J., Eymard, R., Gallouët, T., Herbin, R.: A unified approach to mimetic finite difference, hybrid finite volume and mixed finite volume methods. Math. Models Methods Appl. Sci. 20(2), 265-295 (2010). DOI 10.1142/S0218202510004222. URL http://dx.doi.org/10.1142/S0218202510004222

5. Droniou, J., Eymard, R., Herbin, R.: Gradient schemes: generic tools for the numerical analysis of diffusion equations. M2AN Math. Model. Numer. Anal. 50(3), 749-781 (2016). DOI $10.1051 / \mathrm{m} 2 \mathrm{an} / 2015079$. Special issue - Polyhedral discretization for PDE

6. Eymard, R., Guichard, C.: DGM and GDM (2017). Submitted to FVCA8 